CARNETS DE Carnets de géographes

GÉOGRAPHES.

15 | 2021

Les dimensions spatiales du maintien de l'ordre

\title{
Au-delà des murs. Maintenir l'ordre à la frontière franco-britannique
}

Camille Guenebeaud

\section{OpenEdition}

1 Journals

Édition électronique

URL : https://journals.openedition.org/cdg/7293

DOI : $10.4000 /$ cdg. 7293

ISSN : 2107-7266

Éditeur

UMR 245 - CESSMA

Référence électronique

Camille Guenebeaud, «Au-delà des murs. Maintenir l'ordre à la frontière franco-britannique », Carnets de géographes [En ligne], 15 | 2021, mis en ligne le 30 avril 2021, consulté le 27 mai 2021. URL : http:// journals.openedition.org/cdg/7293; DOI : https://doi.org/10.4000/cdg.7293

Ce document a été généré automatiquement le 27 mai 2021.

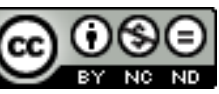

La revue Carnets de géographes est mise à disposition selon les termes de la Licence Creative Commons Attribution - Pas d'Utilisation Commerciale - Pas de Modification 4.0 International. 


\title{
Au-delà des murs. Maintenir l'ordre à la frontière franco-britannique
}

\author{
Camille Guenebeaud
}

\section{Introduction}

En janvier 2019, les ministres français et britannique de l'Intérieur, Christophe Castaner et Sajid David, signent un plan d'action pour renforcer la surveillance de la frontière. Financé par le gouvernement anglais à hauteur de 7 millions d'euros, ce plan succède à une série d'accords conclus depuis près de trente ans qui ont conduit au renforcement et à la militarisation des points de passage vers l'Angleterre. Installation de barbelés, de grillages, de systèmes de vidéo-surveillance : les ports et le site d'entrée du tunnel sous la Manche sont devenus hautement clôturés et compartimentés.

Cette «production du mur » selon les mots de Claude Raffestin, «autrement dit de la frontière matérialisée et bien visible» (Raffestin, 1986), constitue une des figures spatiales les plus souvent associées au maintien de l'ordre en contexte frontalier. Les murs symbolisent avec force les politiques d'immobilisation et de blocage des personnes migrantes tout en participant à la «performance théâtralisée et spectacularisée » (Brown, 2009 : 24) de la fermeture des frontières. Or, ils n'épuisent pas à eux seuls les logiques spatiales du maintien de l'ordre. Cet article propose d'appréhender comment, à la frontière franco-britannique, ces pratiques d'immobilisation des personnes migrantes s'articulent à des dispositifs policiers de déplacements forcés.

La ville de Calais, espace où se concentre la majeure partie du trafic transmanche, est un lieu clé depuis lequel le gouvernement organise une politique de dispersion et d'éloignement des personnes migrantes. En effet, si les pouvoirs publics français participent à empêcher les migrant.es d'aller vers l'Angleterre ${ }^{1}$, ils mettent également en œuvre des stratégies pour les empêcher de rester dans la ville. Nous étudierons, dans un premier temps, ces politiques d'effacement de la présence des personnes migrantes de l'espace calaisien, qui alternent entre formes d'encampement et 
destruction des lieux de vie. Puis, nous étendrons l'analyse aux échelles régionale, nationale et inter-étatique pour appréhender des stratégies de maintien de l'ordre par la dispersion.

En axant le propos sur les déplacements forcés, cet article souhaite contribuer plus largement à l'analyse des dispositifs spatiaux de contrôle frontalier. Si de nombreux travaux ont documenté les enjeux d'une gestion spatiale des migrations par l'encampement (Agier, 2011; Clochard, Gastaut et Schor, 2004), l'emmurement des sites-frontière (Berson, 2011; Brown, 2009; Ritaine, 2009) et l'enfermement (Darley, Lancelevée, Michalon, 2013; Kobelinsky, Makaremi, 2008), il s'agit ici de nourrir des analyses qui ont fait émerger la mobilité comme un outil répressif de maintien de l'ordre en contexte frontalier (Darling, 2016; Gill, 2009; Michalon, 2012 ; Pillant et Tassin, 2015; Tazzioli, 2020; Tyszler et al., 2019).

Aux « tentatives violentes d'immobilisation et de fixation spatiale de catégories entières de populations » (Agier, Bazenguissa-Ganga, Mbembe, 2008) s'articulent en effet des stratégies de mise en mouvement forcée qui enferment les personnes dans des situations d'errance.

S'interroger sur ces formes de maintien de l'ordre aux frontières amène à réfléchir aux acteurs qui conçoivent, dirigent et mettent en œuvre les contrôles migratoires. À l'appui d'une enquête de terrain menée à Calais entre 2010 et 2015, je me concentrerai ici sur l'action des pouvoirs publics et des services de police, même si, par ailleurs, les contrôles sont également pris en charge par divers acteurs privés (Guenebeaud, 2019). Je me fonde en particulier sur vingt entretiens réalisés avec des acteurs institutionnels

Compagnies républicaines de sécurité(CRS) et de la Police aux frontières (PAF) 2 et des policiers des qui ont travaillé à Calais entre 1999 et 2014 et qui ont occupé des postes à différents niveaux de la hiérarchie. Je m'appuie également sur plusieurs mois d'observations menées dans des squats et des campements de la ville et sur une littérature grise abondante (notamment des discours de représentant.es politiques, des rapports émanant d'autorités publiques - Inspection générale de la police nationale, Contrôleur général des lieux de privation de liberté, Cour des comptes, Défenseur des droits -, et des rapports produits par les acteurs du monde associatif - Human Right Watch, No Border, CFDA, Cimade).

\section{Du centre vers la périphérie : des politiques d'effacement}

La ville de Calais, symbole des politiques migratoires répressives franco-britanniques, est depuis près de vingt ans le théâtre de pratiques d'éloignement et de mise à l'écart des personnes migrantes orchestrées par les gouvernements successifs. La stratégie des pouvoirs publics français a consisté à deux reprises à écarter ces personnes du centreville en les regroupant dans deux camps à la périphérie de Calais : le premier, le centre de Sangatte, est ouvert en 1999 par le gouvernement de cohabitation de Lionel Jospin, puis fermé en 2002 ; le second, le bidonville Jules Ferry, est créé en 2015 pendant le mandat socialiste de François Hollande et détruit un an plus tard en 2016. Entre deux et depuis 2016, une politique de destruction des lieux de vie et de harcèlement policier prévaut pour forcer les personnes migrantes à quitter la ville. Nous montrerons ici 
comment la continuité entre ces différentes politiques de destruction et d'encampement réside dans la gestion de l'effacement des migrant.es de l'espace urbain.

\section{CONCENTRER ET ÉLOIgNER DE CALAIS}

«Les sites humanitaires se trouvent aux marges, à l'écart de nos lieux de vie ordinaires. Le regard qui est porté vers eux s'enracine dans un rapport égocentré du type centre-périphérie: il ne s'intéresse aux détails de la vie interne de la périphérie que dans la mesure où ces détails mettent en cause le centre lui-même » (Agier, 2003).

Durant les deux périodes où des camps ont été ouverts par les pouvoirs publics à proximité de Calais, leur localisation a été pensée dans cette dynamique centrepériphérie décrite par Michel Agier. Le 24 septembre 1999, la gestion du premier de ces camps, le Centre d'Hébergement et d'Accueil d'Urgence Humanitaire (CHAUH), est confiée à la Croix-Rouge. Situé dans un hangar d'une ancienne usine d'Eurotunnel à proximité du bourg de la commune de Sangatte, et à neuf kilomètres de Calais, la création de ce lieu répond à une volonté d'éloigner les personnes migrantes du centreville. Cette stratégie de mise à distance est décidée par la préfecture, après de fortes protestations de la mairie de Calais du fait de la présence de campements dans le parc Saint-Pierre, en face de l'hôtel de ville, pendant les mois d'été. Le sous-préfet de l'époque explique :

«Le jour où j'annonce que j'ouvre Sangatte, le maire de Calais frappe des deux mains. J'embête un maire qui lui était opposé politiquement. Et surtout, ce qui commençait à naître à Calais avec les migrants non loin du centre-ville, du jour au lendemain, je les héberge à Sangatte et globalement ils ne passent plus par Calais. C'est bingo. ${ }^{3}$

Pour s'assurer de la présence moindre des personnes migrantes dans Calais, celles-ci sont raccompagnées par la police au centre de Sangatte depuis le port ou depuis le site du tunnel, ce qui vaut aux policier.es le surnom de "Taxi police». Cette mesure « moitié humanitaire, moitié de police » (Carrère, 2003a) conditionne l'hébergement à une forme d'assignation à résidence.

En 2002, le ministre de l'Intérieur de l'époque, Nicolas Sarkozy, décide de la fermeture du centre et impulse une politique de destruction des campements et abris que les personnes tentent de recréer dans la ville de Calais. Cette politique perdure pendant plus de dix ans. Il faudra attendre l'été 2014 pour qu'un revirement s'opère à nouveau dans l'action gouvernementale : la décision est prise d'ouvrir un centre d'accueil, le centre Jules Ferry, qui verra le jour au début de l'année 2015 et dont l'organisation est illustrée sur la figure 1 4.

De façon générale, les conditions d'accueil y sont plus restreintes que celles de la période du centre de Sangatte - un seul repas par jour et pas d'hébergement (à l'exception de 100 places pour les femmes et les enfants) - mais l'ouverture de ce lieu répond à une même logique : regrouper les personnes migrantes en un seul point à

l'écart de la ville. 
Figure 1. «Calais : 20 ans d'(in)hospitalité »

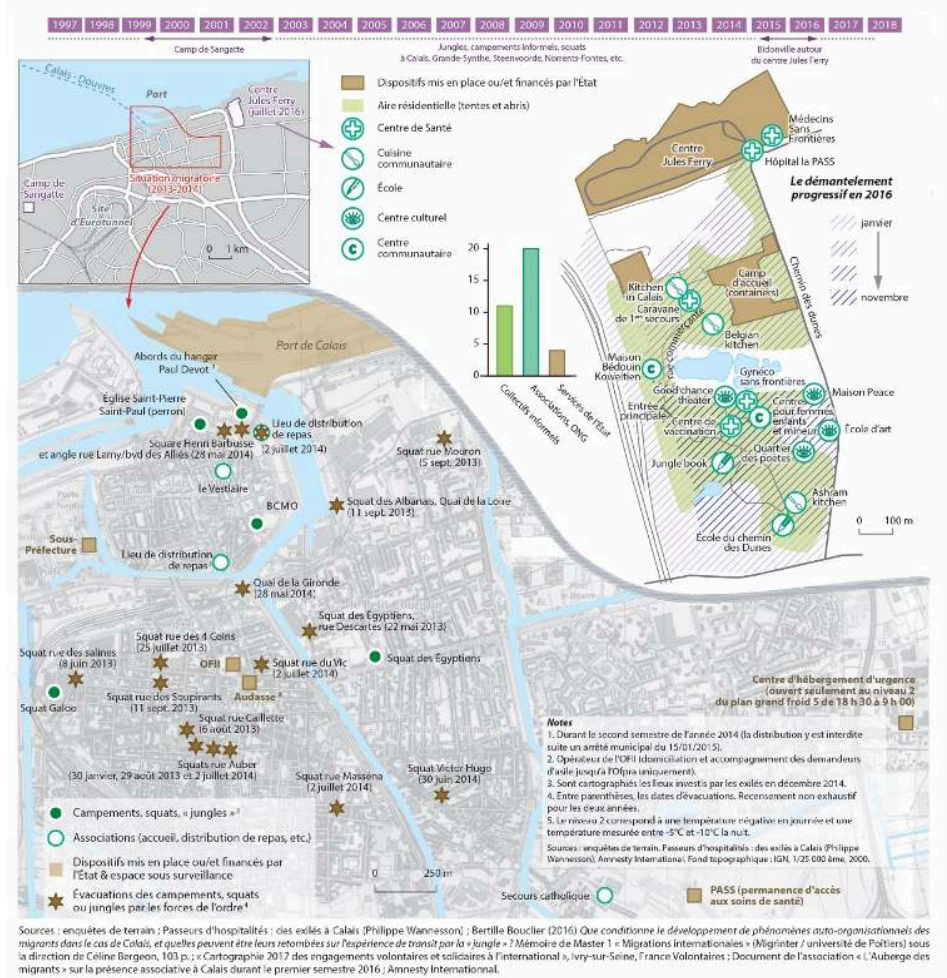

Source : carte réalisée par Olivier Pissoat et Olivier Clochard (Migreurop, 2017 : 153).

Le lieu se situe derrière des grilles qui enserrent une partie de la rocade portuaire, dans une zone de dunes, avec un accès unique vers le centre de Calais. La maire, Natacha Bouchart, annonce que cet espace va permettre de « vider à $80 \%$ la ville de ce phénomène "

5. Comme à la frontière franco-italienne, dans la ville de Vintimille, « décourager les rassemblements de migrants dans l'espace urbain et encourager leur déplacement vers le camp devient un objectif de gouvernement local » (Trucco, $2018: 156)$.

Pour ce faire, 1

es pouvoirs publics poussent les personnes migrantes à quitter les squats du centreville et à s'installer dans la zone de dunes autour du centre :

«C'est dans cette perspective que la préfète a indiqué avoir pris contact avec les représentants de la plupart des associations de soutien aux exilés implantées localement pour leur demander d'inciter les migrants installés dans les différents squats de la ville à s'établir sur le terrain situé à proximité du centre d'accueil Jules Ferry, tout en leur faisant savoir que les jugements d'expulsion portant sur la majeure partie des squats ou "jungles" alors habités étaient susceptibles d'être exécutés. $»^{6}$

Les associations vont être profondément partagées sur ce que la préfecture nomme un "déménagement $»^{7}$ mais qui se fait sous la menace de l'expulsion. Progressivement, tous les squats et campements sont évacués et un camp se forme autour du centre Jules-Ferry.

Ces deux périodes d'encampement organisé par l'État à la périphérie de Calais sont également liées à une autre stratégie d'éloignement: une politique de déguerpissement ${ }^{8}$ qui se déroule de 2002 à 2015 (puis à nouveau à partir de 2016). 


\section{DÉTRUIRE POUR FAIRE PARTIR}

La fermeture du centre de Sangatte ouvre une période durant laquelle les personnes migrantes sont sans cesse contrôlées et chassées de Calais (Carrère, 2003b). Malgré l'apparition progressive de campements et de squats qui se stabilisent dans la durée, comme le camp pachtoune dans la zone des dunes, c'est une stratégie policière de contrôle de la population migrante et de déguerpissement qui prévaut. Le bulldozer, machine régulièrement employée à Calais pour les destructions des lieux de vie, est devenu l'emblème de cette politique.

Ces destructions se font après des temps d'occupation très variables, de quelques jours à plusieurs mois, voire années, en fonction des procédures enclenchées par les pouvoirs publics et les propriétaires des terrains. Mais progressivement au fil des années, tous les grands squats sont démolis : le 24 octobre 2006, c'est la destruction du squat de la Socarenam ${ }^{9}$ quai de la Moselle qui regroupe des Somalien.nes, Soudanais.es, Érythréen.nes et Éthiopien.nes. Ces dernier.es occupent par la suite l'ancienne usine de menuiserie Pagniez, renommée "Africa House ", qui sera détruite en juin 2010. Ils investissent alors l'ancienne usine de dentelle Noyon, située juste à côté, ainsi que l'ancienne usine Thélu, qui seront toutes les deux évacuées et démolies à la fin de l'année 2011. Des personnes occupent également les ruines de l'ancienne usine Darquer mais cet espace est détruit en mai 2012 à l'exception de la façade. D'autres installent une "Africa House » dans d'anciens bâtiments de l'université du Littoral Côte d'Opale qui sont évacués en mars 2012 en vue d'être rasés pour faire place à un éco-quartier. D'autres squats se succèdent ainsi jusqu'en 2015, sans destructions, mais avec des expulsions répertoriées sur la figure 1. Certains d'entre eux sont murés, ainsi que beaucoup d'autres maisons vides, garages, anciens commerces ou usines désaffectées, afin de faire en sorte que les personnes migrantes ne puissent plus s'y installer.

Les campements, situés à l'écart de la ville sont également régulièrement détruits par les forces de police. L'exemple le plus médiatisé est celui du bidonville pachtoune rasé en septembre

2009.

Le lieutenant François P., qui fait partie des compagnies de CRS qui participent à l'évacuation du lieu, explique comment l'espace naturel a été détruit par ces bulldozers :

« La jungle je l'ai vu rasée, j'ai vu le désert. Je pensais pas qu'ils feraient ça [...]. Ça a même dévasté la dune, il y avait plus rien. Moi qui aime beaucoup la mer, ça me faisait mal au cœur d'abîmer cette dune. Ils ont gratté le sable, ils ont pas enlevé que les petites tentes, ils ont tout pillé. Ils ont enlevé toute la végétation. $»^{10}$

La destruction a pour objectif de rendre l'espace impropre à tout réinvestissement, à toute réappropriation possible. Couper les arbres, qui sont un appui à la construction d'abris et qui protègent du soleil et de la pluie, participe de cette stratégie.

En dehors de ce moment de destruction, les squats et les campements ne sont pas, dans la majorité des cas, des lieux privés soustraits au contrôle. L'action policière s'y maintient pour contraindre et limiter l'inscription spatiale des personnes migrantes dans la ville. Au-delà du recueil d'informations (localisation des camps, recensement du nombre de personnes), les policier.es réalisent régulièrement - voire quotidiennement des intrusions et des interpellations dans ces espaces. Ces contrôles sont le fait de la brigade de la voie publique de la PAF qui est chargée de l'identification et de la surveillance des squats et qui est appuyée par l'action des compagnies de CRS. En 2010, 
lors de périodes d'observations menées à Calais en restant vivre au quotidien dans des squats et des campements du centre-ville, je prends note de ces interventions à répétition.

«La police vient fréquemment à l'“Africa House”, surtout le matin, et arrête à chaque fois de nombreuses personnes. La semaine dernière j'ai assisté à une expulsion organisée à l'appui de dix cars de CRS vers sept heures. Quelqu'un a sifflé pour prévenir de l'arrivée des policiers. Les personnes déjà réveillées ont couru prévenir les autres et ont tenté de se cacher dans les multiples recoins du lieu. Les policier.es ont maintenu à l'écart les quelques personnes qu'ils identifiaient comme des militant.es, dont moi, et ont arrêté celles qu'ils identifiaient comme des migrant.es. Ils ont rempli les cars et sont repartis. $»^{11}$

Le rapport associatif, Calais deux ans après, propose une chronologie sur ces multiples intrusions sur les lieux de vie entre novembre 2011 et juin 2014. Ce rapport souligne notamment les arrestations très régulières faites dans le camp afghan :

«Ainsi, selon nombre de personnes de nationalité afghane vivant dans les jungles, les forces de l'ordre ont pris l'habitude de se rendre sur leur lieu de vie au moins trois fois par semaine, le plus souvent le lundi, le mercredi et le vendredi, aux alentours de 8 heures. Chaque visite se solde par un contrôle d'identité et des arrestations. $»^{12}$

Parfois, ces intrusions prennent la forme d'expulsions de fait sans procédure juridique. C'est le cas lorsque le campement est vidé et que, pendant que les personnes sont conduites à l'hôtel de police, les affaires pour dormir (tentes, sacs de couchage) sont emmenées ou rendues inutilisables. Un policier d'une compagnie de CRS décrit dans un entretien de 2014 cette procédure hors de tout cadre réglementaire qui vise à empêcher les personnes migrantes de se réinstaller : "Avec une compagnie, on avançait et on cassait les tentes. Comme ça ils ne pouvaient plus habiter là ».

Ces « visites répétées sur des lieux de vie, à toute heure du jour et de la nuit $»^{13}$ visent à rendre impossible l'établissement d'un quotidien. La destruction des ustensiles de cuisine par les forces de l'ordre lors de ces interventions est relevée à plusieurs reprises par des acteurs associatifs entre 2009 et 2011. Du gaz lacrymogène est ponctuellement répandu sur les réserves de nourriture et sur l'unique point d'eau du bidonville pachtoune ${ }^{14}$. Cette entrave au fait de pouvoir se nourrir est une stratégie qui joue sur la limitation d'une ressource biologique nécessaire. Les interventions durant le sommeil sont une autre de ces ressources. À plusieurs reprises, les rapports des associations relèvent les faits suivants : arrivée des forces de l'ordre tard le soir ou très tôt le matin, plein phares, coups de klaxon pour réveiller les personnes migrantes qui ne tentent pas le passage cette nuit-là. Dans un entretien mené avec un policier en service avant 2009, celui-ci décrit ces réveils volontaires tôt le matin : « Quand on allait dans le bois à cinq heures du matin pour les réveiller, on en prenait 40 en 10 minutes ".

Lors de ces interventions, en particulier dans les lieux de vie retirés loin du centre-ville, des violences physiques de la part des policier.es sont rapportées par certaines personnes migrantes ${ }^{15}$.

Ces violences sont dénoncées régulièrement par le milieu associatif depuis la période du centre de Sangatte. Dans les discours, une distinction revient entre les CRS et la PAF,

l'action des premiers étant jugée plus violente et incertaine que celle des seconds. Les associations affirment par exemple que les compagnies de CRS, qui - souvent mais pas

toujours - viennent d'arriver et ne connaissent pas encore la réglementation, ne respectent pas un accord passé avec les autorités qui sanctuarise la distribution des repas de toute interp 
ellation. Le commandant Jacques

T. confirme, en entretien, qu'il est nécessaire de briefer les nouvelles compagnies qui arrivent sur Calais pour éviter que, dans une optique de faire du chiffre, elles se rendent au lieu de distribution et fassent des arrestations massives

${ }^{16}$.

Ces pratiques policières, décrites dans de nombreux documents associatifs, ainsi que dans différents rapports du Défenseur des droits ${ }^{17}$, sont à plusieurs reprises déniées par les pouvoirs publics. Le ministère de l'Intérieur, en réponse à une décision du Défenseur des droits de 2012 (qui atteste d'expulsions de campements réalisées en dehors de tout cadre juridique, de destructions d'effets personnels, et de " comportements individuels consistant à provoquer ou humilier les migrants ${ }^{18}$ ), parle de faits reposant "essentiellement sur des déclarations de responsables d'associations rapportant des propos non vérifiables et concernant des faits anciens qu'aucun élément objectif ne peut soutenir aujourd'hui $»^{19}$. En 2015, un rapport mené à Calais par Jean Aribaud et Jérôme Vignon et commandé par le même ministère fait mention de potentielles violences policières non attestées ${ }^{20}$. En 2017, un rapport des Inspections générales de la police nationale, de l'administration et de la gendarmerie nationale va plus loin et considère comme " plausibles des manquements à la doctrine d'emploi de la force et à la déontologie policière, principalement à Calais. Ils portent sur des faits de violences, sur un usage disproportionné des aérosols lacrymogènes, la destruction d'affaires appartenant aux migrants $»^{21}$. Cependant, la communication politique prend le contre-pied du rapport. En 2018, à l'occasion d'un discours devant les forces de l'ordre à Calais, Emmanuel Macron déclare : «Je ne peux pas laisser accréditer l'idée que les forces de l'ordre exercent des violences physiques, confisquent des effets personnels, réveillent les personnes en pleine nuit, utilisent du gaz lacrymogène sur des points d'eau ou au moment de la distribution des repas. Si cela est fait, c'est contraire à toute déontologie $\aleph^{22}$.

La question du respect ou du non-respect de la déontologie dans les pratiques policières à Calais s'articule ici à une dimension plus structurelle d'utilisation de la « violence comme politique » (Alaux, 2013). La destruction des abris et des affaires personnelles représente en effet la matérialisation d'une stratégie politique plus large décrite ainsi parun ancien sous-préfet : « Moi, on m'avait dit "il faut leur rendre la vie impossible pour qu'ils en aient marre et qu'ils s'en aillent" $»^{23}$.

Nommée « stratégie d'auto-expulsion » par Éric Fassin à propos de la gestion politique et spatiale des Roms, cette politique " suppose de créer des conditions insupportables pour ceux que l'on prétend faire partir d'eux-mêmes. » (Fassin et al,2014:45).

A Calais, cette stratégie marque un ordre territorial qui exclut les migrant.es et vise à les éloigner progressivement de la ville, de la frontière et de l'espace national.

\section{Gouverner par la dispersion}

La logique centre-périphérie qui s'applique aux politiques de concentrationéloignement et de déguerpissement des personnes migrantes hors de la ville de Calais, s'articule également aux échelles régionale, nationale et inter-étatique. En observant l'évolution des stratégies policières, on voit se dessiner une forme de gouvernement 
par la dispersion - en particulier entre 2002 et 2015 et depuis 2016 - selon trois modalités: expulsion hors du territoire national, déplacement dans d'autres départements français et dispersion au sein de la région.

\section{EXPULSER HORS DE L'ESPACE NATIONAL}

La stratégie la plus classique est celle de l'expulsion, qui vise à soustraire certaines personnes à l'espace national en les éloignant vers leurs pays d'origine ou en les dispersant dans différents pays européens. Cette stratégie s'organise à Calais depuis le centre de rétention administrative (CRA) de Coquelles. En service depuis 2003, cet espace permet de maintenir enfermée une personne qui fait l'objet d'une décision d'éloignement en vue de préparer son expulsion. L'aménagement du lieu a été pensé pour favoriser le confinement et l'expulsion à l'abri des regards. Situé au sein de l'hôtel de police de la PAF, lui-même accolé au site d'Eurotunnel, le CRA est très peu visible de l'extérieur. Il n'est d'ailleurs pas indiqué depuis l'entrée. Cette euphémisation architecturale de l'enfermement relève d'un parti pris comme le montre cet extrait du permis de construire :

«Le CRA s'implante relativement éloigné de l'emprise publique. La zone d'hébergement et les espaces extérieurs de détente lui tournant le dos (...) l'aspect extérieur du projet linéaire et bas s'accrochant aux courbes de niveaux a pour but non pas de se montrer mais de se fondre dans le paysage. $»^{24}$

Cette «fonte » dans le paysage se fait en utilisant le dénivelé du terrain. Les grillages qui entourent le CRA pour délimiter et empêcher l'accès au site d'Eurotunnel ne sont visibles que de l'intérieur. Des talus artificiels limitent la vue depuis l'extérieur et il a été planté «un alignement d'arbres côté Sud, afin de protéger la vue du bâtiment depuis l'accès général au site ${ }^{25}$.

$\mathrm{Au}$ sein de l'enceinte de police, le centre de rétention fait face à une annexe du tribunal de grande instance (TGI) de Boulogne qui participe à faciliter des procédures d'expulsion ${ }^{26}$. L'annexe a l'avantage pour l'administration de limiter les frais en personnels liés aux multiples escortes nécessaires entre Calais et le TGI de Boulogne. Dans un même temps, elle permet selon Vincent M., officier de police judiciaire, de limiter la visibilité publique des audiences :

«La justice a dû s'adapter en autorisant les salles d'audience à proximité des centres de rétention. En termes financiers, c'était quand même nettement plus simple. Et puis amener des personnes dans une audience ouverte à tout le monde à Boulogne... Même si, ici aussi, les audiences sont ouvertes à tout le monde, c'est la loi. Mais ici vous avez rarement un public extérieur, alors que dans un TGI vous avez toujours des gens. $»^{27}$

Effectivement, très peu de personnes viennent assister aux audiences dans l'annexe de Coquelles. Elle est d'ailleurs peu visible: seule une plaque «Annexe du tribunal de grande instance » indique l'entrée sur le côté gauche de l'hôtel de police.

Le rapprochement géographique entre le lieu de rétention et le lieu de jugement impacte les conditions de mise en œuvre de la justice ${ }^{28}$. Certaines personnes migrantes parlent du «juge de la police», soulignant la confusion des rôles engendrée par la proximité spatiale. Des avocat.es ont également questionné l'indépendance des interprètes du fait de leur présence quotidienne dans un même lieu que les policier.es, conduisant à des réseaux d'inter-connaissances ${ }^{29}$. Pourtant, c'est dans cette salle qu'ont 
lieu les audiences du TGI depuis 2005 et c'est ici que se décide, en l'absence de regard extérieur, la libération ou la reconduite de la rétention des personnes migrantes.

Depuis 2006, 500 à 1500 personnes sont expulsées chaque année après avoir été retenues au centre de rétention de Coquelles $^{30}$. Ces chiffres ne doivent cependant pas masquer une réalité majeure dans la situation calaisienne: une grande partie des personnes présentes sur le littoral viennent de pays en guerre et ne sont donc pas, selon la Convention de Genève du 28 juillet 1951, expulsables. En 2008, seules 4,2 \% des interpellations ont conduit à des expulsions ${ }^{31}$. Bernard S., policier à la PAF, décrit cette situation :

«Il y a quand même énormément de pays où l'on ne peut pas reconduire les gens. Il faut que les consulats les acceptent. Et après il $\mathrm{y}$ a les pays où les gens que l'on reconduirait seraient dans des situations politiques trop dangereuses, donc on ne peut pas les reconduire et c'est quand même le problème de Calais. Sur Calais, les Syriens vous ne pouvez pas les reconduire. Les Afghans on pouvait théoriquement les reconduire mais c'est très compliqué. La corne de l'Afrique c'est impossible à reconduire. Donc les reconduites frontières sont très compliquées. »

Il est particulièrement marquant de constater qu'un grand nombre de personnes expulsées le sont dans le cadre des procédures Dublin ${ }^{32}$ ou Schengen ${ }^{33}$. La majorité des expulsions concernent donc le transfert vers d'autres pays européens et non pas le retour vers le pays d'origine. C'est ce que les associations intervenant dans les centres de rétention nomment le "grand détournement » dans leur rapport de $2014: 55 \%$ des personnes éloignées en 2014 l'ont été vers un pays de l'Union Européenne ${ }^{34}$. À Coquelles ce taux varie entre $55 \%$ et $85 \%$ depuis 2006 (figure 2). Ces expulsions permettent de remplir les objectifs chiffrés de la politique d'immigration en éloignant des personnes de certaines nationalités vers d'autres pays européens faute de pouvoir les renvoyer dans leur pays d'origine.

Figure 2. Les expulsions depuis Calais : des renvois avant tout en Europe ?

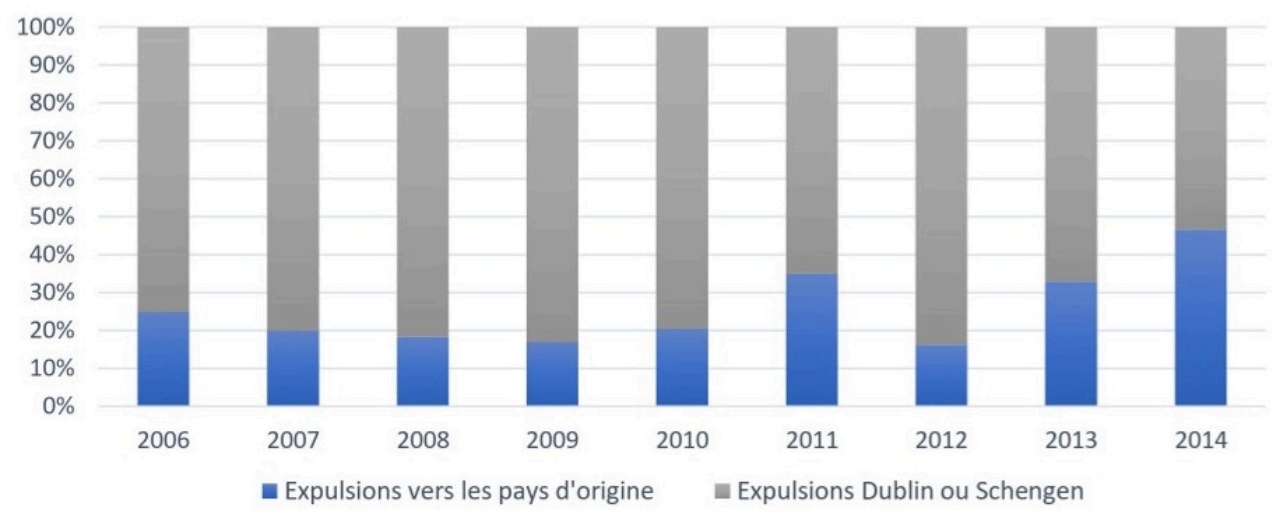

Source : "Centres et locaux de rétention administrative », Rapports 2006-2014, Cimade, Forum des réfugiés, Ordre de Malte, Assfam.

A Calais, les expulsions réalisées vers les pays d'origine restent donc très peu nombreuses. Cette politique d'éloignement étant peu effective, les pouvoirs publics français ont développé parallèlement des stratégies de dispersion à d'autres échelles. 


\section{ÉLOIgNER DU PAS-DE-CALAIS}

En décembre 2002, le ministère de l'Intérieur adresse un télégramme au préfet du Pasde-Calais intitulé "Éloignement des étrangers en séjour irrégulier non éloignables présents aux abords de Sangatte" (Hénocq, 2003). Ce télégramme annonce une politique qui sera menée pendant plusieurs années, à savoir trouver des stratégies relatives à l'éloignement de Calais de personnes qui ne peuvent pas être expulsées hors du territoire national.

Dans le contexte qui suit la fermeture du centre de Sangatte et qui est marqué par la répression permanente de toute tentative d'installation des personnes migrantes dans la région, une série d'opérations baptisées Ulysse sont mises en place. Leur objectif est de déplacer les migrant.es dans d'autres régions en passant par l'hébergement: les personnes non expulsables sont ainsi conduites au sein de Centres d'Hébergement et de Réinsertion sociale (CHRS), de Centres d'Accueil pour Demandeurs d'Asile (CADA), de foyers ou parfois simplement dans des hôtels dans d'autres départements (Clochard, 2007 : 293). Des effectifs de police, notamment des CRS, effectuent les escortes. Le coût de ces opérations est évalué à 600000 euros par an ${ }^{35}$.

En 2006, le comité interministériel de contrôle de l'immigration poursuit cette action avec un dispositif rebaptisé Ulysse 3. Les personnes migrantes interpellées et non expulsables sont déplacées dans ces centres d'hébergement dans différentes régions de France où leur est proposé une aide au retour pour un départ "volontaire » ou un transfert pour demander l'asile en CADA loin de Calais, incitant provisoirement à la demande d'asile en France pour mieux les éloigner du Pas-

de-Calais.

Celles et ceux qui refusent se voient notifier un arrêté de reconduite à la frontière (APRF) et des arrestations ont parfois lieu à la sortie des centres d'hébergement avec des placements en centre de rétention administrative ${ }^{36}$.

Cependant, les différents entretiens menés avec des policiers de la PAF et des CRS soulignent l'inefficacité globale de ce système, car la plupart des personnes reviennent très rapidement à Calais. C'est ce que décrit ici un lieutenant de CRS :

«On les emmenait dans l'Est, en Bretagne ou dans le Sud. C'était pas vraiment les remettre à la sortie de la frontière. On les sortait juste de la ville. On les éloignait. On partait en bus avec un véhicule devant et un véhicule derrière pour pouvoir les surveiller et on les déposait là-bas. Mais eux, ils revenaient. [...] Moi je me souviens, une fois, j'avais amené une dizaine de migrants dans un foyer à Metz et quand je suis rentré, ils étaient déjà revenus avant moi. C'était un cercle vicieux. Ça a été abandonné au bout de quelques années parce que c'était une fortune sur le budget administratif: presque un million d'euros pour des résultats très peu convaincants. $»^{37}$

Cette stratégie de déplacement contraint par l'hébergement réapparaît en octobre 2015, lorsque le ministère de l'Intérieur crée les Centres d'Accueil et d'Orientation" (CAO). L'objectif est alors de maîtriser et faire diminuer autant que faire se peut, avant l'entrée dans la période hivernale, la population du campement "

${ }^{38}$ Jules Ferry à Calais où vivent près de 6000 personnes. Les personnes migrantes sont fortement incitées, puis contraintes, à rejoindre ces centres situés dans des régions éloignées de Calais. Ces lieux proposent une mise à l'abri provisoire sans que cela soit 
conditionné à une demande d'asile si les personnes s'engagent à renoncer à rejoindre l'Angleterre.

Cesstratégies d'éloignement de Calais par l'hébergement - qui ont per

duré depuis le début des années 2000 et qui continuent encore aujourd'hui - participent d'un dispositif plus vaste, qui comprend également l'éloignement par le placement en rétention.

Alors que la rétention est strictement prévue en vue d'organiser l'expulsion de l'étranger.e, cette procédure est parfois utilisée afin de «vider » la ville des personnes migrantes et de les éloigner de la région. Cette pratique, qui a cours depuis la fermeture du centre de Sangatte, s'apparente à un détournement de procédure. Elle consiste à placer dans des centres de rétention administrative d'autres régions des personnes qui ne sont pas expulsables, ce afin de les éloigner provisoirement de Calais.

C'est le cas en décembre 2002 d'une cinquantaine de Kurdes irakien.nes, interpellé.es dans le Pas-de-Calais, qui sont transféré.es dans des CRA de la région parisienne avant d'être placé.es dans des foyers (Hénocq, 2003). C'est également la situation de 129 personnes placées en rétention suite à la destruction d'un camp pachtoune en septembre 2009. Aucune d'entre elles n'est retenue au CRA de Coquelles ou de Lille, mais elles sont toutes transférées dans les CRA de Toulouse, Paris, Marseille, Nice, Lyon ou encore Nîmes.

En novembre 2015, le Contrôleur général des lieux de privation de liberté (CGLPL) décrit un système comparable ${ }^{39}$. Il met à jour un dispositif de transfert de migrant.es dans des CRA éloignés du Calaisis alors que le CRA de Coquelles n'est pas complet. C'est à cette période également que la presse révèle l'utilisation d'un jet privé loué par l'État à hauteur d'un million et demi d'euros pour l'année 2015 afin de reconduire depuis l'aérodrome de Marck des personnes dans les CRA de Toulouse, Metz, Nîmes, Hendaye ou encore Perpignan ${ }^{40}$.

«Il est donc établi que, depuis le 21 octobre 2015, le placement en rétention administrative est utilisé non pas aux fins d'organiser le retour dans les pays d'origine mais dans le seul objectif de déplacer plusieurs centaines de personnes interpellées à Calais et de les répartir sur l'ensemble du territoire français, et ce dans le but de "désengorger" Calais. Il s'agit là d'une utilisation détournée de la procédure qui entraîne des atteintes graves aux droits fondamentaux des personnes ainsi privées de liberté. Le CGLPL recommande qu'il y soit mis fin. $»^{41}$

Ces différentes opérations qui déplacent les personnes sur tout le territoire participent à des stratégies qui se retrouvent à d'autres frontières européennes. C'est le cas à la frontière franco-italienne où le gouvernement italien opère des déplacements forcés en bus vers le hotspot de Tarente à 1200 kilomètres de là (Tazzioli, 2020). Dans la région de Nador au nord du Maroc, près de l'enclave espagnole de Melilla, les forces de police marocaines procèdent à des arrestations massives dans les campements et déplacent les personnes en bus vers le sud du pays, le plus loin possible de l'entrée de l'espace Schengen (Tyszler et al. 2019). À Calais, à la sortie de cet espace qui pour nombre de migrant.es correspond à la sortie de l'espace Schengen, ces pratiques d'éloignement se combinent à des stratégies locales de dispersion des personnes présentes dans la région. 


\section{DISPERSER DANS LA RÉgION}

Afin de rendre moins visibles les personnes migrantes à la frontière francobritannique, la stratégie gouvernementale qui a cours de 2002 à 2015 vise à leur dispersion dans la région. La fermeture du centre de Sangatte marque le début de cette politique.

Tout d'abord, les services de police procèdent à des éloignements de personnes dans la campagne environnante. Certain.es migrant.es sont ainsi déposé.es par la police à plusieurs dizaines de kilomètres de Calais, d'autres reçoivent des sauf-conduits sans fondement juridique comportant la mention : "ne doit pas se trouver à 60 kilomètres du littoral dans les quarante-huit heures suivant la notification» (Hénocq, 2003). Ces pratiques participent à une pression plus vaste mise sur les personnes migrantes pour qu'elles se dispersent autour de Calais, comme le souligne Jérôme F., policier de la PAF :

«Il y a une nécessité d'éviter que ça stagne trop sur Calais. Le but c'est de les disperser. [...] Il y a vraiment cette pression qui a un but, c'est entre guillemets de faire partir les migrants ailleurs que sur Calais, les repousser. Pas les faire repartir chez eux mais vraiment les repousser de Calais. Ça a marché pendant un temps [...]. La stratégie qui a été mise en place par la PAF a fonctionné, les migrants se sont retrouvés sur Dunkerque et sur les extérieurs. $\$^{42}$

D'autres policiers décrivent l'objectif « de maintenir un bas niveau sur le Calaisis ${ }^{43}$ ou de «circonscrire cette population $»^{44}$ en dehors de Calais. Jérôme F. témoigne d'une stratégie qui fonctionne et qui pousse les migrant.es à s'installer aux alentours de la ville jusqu'en 2008-2009, période où le nombre de personnes présentes dans le camp pachtoune de Calais augmente. En septembre 2009, ce camp est détruit et les associations dénoncent le fait que cela va continuer à disperser les migrant.es dans la région. La maire de Calais, Natacha Bouchart, appuie cette dispersion :

«Mais déplacer le problème, ça me va très bien! Après tout ce qu'on subit depuis huit ans! [...] Au moins, déplacer les problèmes, ça nous soulagera pendant quelques mois ou quelques années. Ce ne sera plus chez nous. D’autres le subiront à leur tour. ${ }^{45}$

Si ces stratégies policières de dispersion des personnes migrantes loin de Calais, amorcées en 2002 et qui se sont poursuivies dans les années qui ont suivi, n'ont pas empêché la persistance de campements dans la ville, elles ont parallèlement conduit à une nouvelle géographie du passage, avec une multiplication des points de traversée.

« La multiplication des campements informels sur l'ensemble des régions littorales de la Manche et de la Mer du Nord constitue l'une des principales conséquences de la fermeture du camp de Sangatte et du durcissement des contrôles migratoires qui l'ont suivie. Les exilés qui y vivent sont confrontés à différentes formes de mise à l'écart, à des conditions matérielles déplorables et à des contrôles répétés par les forces de police. Cette dispersion rend le phénomène moins visible, ce qui a permis aux pouvoirs publics de laisser croire que ces étrangers avaient peu ou prou disparu et que la question était pour l'essentiel réglée. $»^{46}$

Le passage s'étend désormais sur le littoral de Roscoff à Dunkerque mais aussi jusqu'en Belgique. Des campements apparaissent à proximité des ports transmanches. À Ouistreham, Dieppe, Cherbourg et Dunkerque notamment, la présence des personnes migrantes s'est inscrite dans la durée. Des campements se sont également établis au fil des ans le long des autoroutes qui relient Paris à Dunkerque ou à Calais, à proximité des aires de repos. Sur l'A25 se trouve le camp de Steenvoorde, proche de l'aire de SaintLaurent. Sur l'A26 en direction de Calais, de nombreux campements ont été créés : près 
de Norrent-Fontes, de Saint-Omer ou encore de Angres. Ces nouveaux camps sont le résultat des politiques de dispersion, mais aussi, en réaction, de stratégies des personnes migrantes d'implantation dans des espaces plus éloignés de la répression qui se déploie à Calais.

Dans ces espaces, les logiques spatiales du maintien de l'ordre sont redéfinies, en particulier parce que les camps sont souvent dans le périmètre d'action de la gendarmerie et moins investis par la PAF et les CRS que dans la ville de Calais. Dans un entretien mené en 2014 avec le maire de Norrent Fontes, une commune rurale de 1500 habitants située à proximité d'un campement, celui-ci fait le récit d'un "épisode épique » survenu entre la PAF et la gendarmerie. Après que la PAF soit intervenue sur le parking de «façon assez violente ", plusieurs personnes étant blessées, l'association Terre d'Errance propose aux migrant.es d'aller porter plainte à la gendarmerie. Le surlendemain, la PAF se rend sur le campement pour faire une enquête. Le maire reçoit alors un appel du sous-préfet lui demandant d'aller sur place avec la gendarmerie pour protéger les potentiels témoins de l'agression et empêcher la PAF de procéder à des arrestations : "Et donc on l'a fait, avec le capitaine de gendarmerie qui était là, la gendarmerie a pris les personnes que la PAF recherchait ». Au-delà de cet épisode singulier, l'appartenance à différents services de police - PAF, Gendarmerie, CRS -, qui implique un rapport et un ancrage au territoire différent, jouent sur les modalités d'application des stratégies policières.

En observant ces stratégies, nous avons voulu montrer ici comment, en utilisant "l'éloignement comme ressource ${ }^{47}$, les pouvoirs publics travaillent à disperser les personnes migrantes à différentes échelles, depuis l'expulsion hors du territoire national jusqu'à l'éloignement temporaire du centre-ville calaisien.

\section{Conclusion}

Porter l'analyse au-delà des murs établis aux frontières permet de sortir de l'image de la frontière-ligne et de se décentrer des politiques de blocage des migrations, pour comprendre comment elles s'articulent à des stratégies de mobilités forcées. À la frontière franco-britannique, si les pouvoirs publics français ont participé à la bunkerisation des points de passage vers l'Angleterre, ils ont également orchestré une politique de mise à l'écart et d'éloignement des personnes migrantes de Calais. Les pratiques d'expulsion hors du territoire national, de déplacement hors de la région, de dispersion sur le littoral, ainsi que les stratégies de harcèlement policier qui visent à forcer les personnes à partir d'elles-mêmes sont autant d'éléments dont la continuité se trouve dans cette gestion de leur éloignement et de leur invisibilité à la frontière.

Face à ce refus matériel de leur inscription spatiale et à la mise en scène symbolique de leur disparition, « l'absence attendue de l'étranger est contredite par sa signature, qui

jaillit dans la nation même, à l'intérieur du groupe de référence qui veut pourtant

l'exclure » (Blanc, $2010: 1$

54).ÀCalais, 1

es personnes migrantes continuent de marquer la ville de leur présence. Malgré les expulsions très régulières, des lieux de vie, des squats et des campements sont reconstruits en permanence. « Destroying ours houses will not make us disappear 
» est un message qui revient sur des banderoles lors de plusieurs expulsions, affirmant droit à l'espace

face à des politiques qui créent partout des espaces de soustraction (Kobelinsky et Makaremi, 2009 : 259).

Ces formes de résistance déconstruisent la logique de spectacularisation du pouvoir politique qui, par la dispersion et l'éloignement des personnes migrantes, met en scène leur disparition à la frontière. Pour autant, elles ne signifient pas l'absence d'impact de ce pouvoir sur les conditions de vie.

Les déplacements contraints dans et hors du territoire national participent à assigner dansousurla frontière(Balibar, $2001:$ 192).

les personnes migrantes à vivre L'errance est une des caractéristiques de cette condition migrante et une des conséquences des politiques gouvernementales menées depuis plus de 20 ans. Elle symbolise«

le caractère multiforme que peut prendre le concept de mise à l'écart des étrangers par les sociétés européennes, sans qu'il soit besoin de barreaux ni de murs pour entraver leur circulation » (Clochard, Gastaut, Schor, 2004). Ce vécu est celui des camps,des mobilités forcées, des espaces d'entre-deux marqués par la précarité, en attente d'être accueillis.

\section{BIBLIOGRAPHIE}

AGIER M. (2011), « L'encampement du monde », Plein droit, vol. 90, no. 3, pp. 21-24.

AGIER M. (2003), « La main gauche de l'Empire », Multitudes, no. 1, pp. 67-77.

AGIER M., BAZNGUISSA-GANGA R., MBEMBE A. (2008), « Mobilités africaines, racisme français », Vacarme, vol. 2, no. 43, pp. 83-85.

ALAUX J.P. (2013), « À Calais, la violence comme politique », Plein droit, vol. 97, no. 2, pp. 28-31.

BALIBAR E. (2001), Nous, citoyens d'Europe? Les frontières, l'État, le peuple, Paris, La Découverte.

BERSON E. (2011), « Un mur sur le détroit du Pas de Calais », Recueil Alexandries, Collections Esquisses, no. 23 [En ligne], URL : http://www.reseau-terra.eu/article1191.html.

BLANC G. Le (2010), Dedans, dehors La condition d'étranger, Paris, Seuil.

BLOT J., SPIRE A. (2014), « Déguerpissements et conflits autour des légitimités citadines dans les villes du Sud », L'Espace Politique, vol. 22, no. 1 [En ligne], URL : http://espacepolitique.revues.org/ 2893.

BROWN W. (2009), Murs. Les murs de séparation et le déclin de la souveraineté étatique, traduit par Nicolas Vieillescazes, Paris, Les Prairies Ordinaires.

CARRERE V. (2003a), « Sangatte, un symbole d'impuissance », Plein droit, no. 58, pp. 4-8. 
CARRERE V. (2003b), « Sangatte : trois ans de leurre, une fin en trompe-l'oeil », Vacarme, no. 22, pp. 52-56.

CLOCHARD O. (2007), Le jeu des frontières dans l'accès au statut de réfugié - Une géographie des politiques européennes d'asile et d'immigration, Université de Poitiers, $487 \mathrm{p}$.

CLOCHARD O., GASTAUT Y., SCHOR R. (2004), « Les camps d'étrangers depuis 1938 : continuité et adaptations ", Revue européenne des migrations internationales, vol. 20, no. 2, pp. 57-87.

DARLEY M., LANCELEVEE C., MICHALON B. (2013), « Où sont les murs? Penser l'enfermement en sciences sociales ", Cultures \& Conflits, no. 90, pp. 7-20.

DARLING J. (2016), « Asylum in Austere Times: Instability, Privatization and Experimentation within the UK Asylum Dispersal System », Journal of Refugee Studies, Vol. 29, no. 4, pp. 483-505.

FASSIN É., FOUTEAU C., GUICHARD S., WINDELS A., (2014), Roms et riverains. Une politique municipale de la race, Paris, La Fabrique éditions.

GILL N. (2009), « Governmental mobility: The power effects of the movement of detained asylum seekers around Britain's detention estate », Political Geography, no. 28, pp. 186-196.

GUENEBEAUD C. (2019), « "Nous ne sommes pas des passeurs de migrants" : le rôle des transporteurs routiers et maritimes dans la mise en œuvre des contrôles à la frontière francobritannique », Lien social et Politiques, no. 83, pp. 103-122.

HENOCQ M. (2003), « Bricolages administratifs », Plein droit, no. 58, pp. 29-32.

KOBELINSKY C., MAKAREMI C. (2008), « Editorial. Confinement des étrangers : entre circulation et enfermement », Cultures \& Conflits, no. 71, pp. 7-11.

KOBELINSKY C., MAKAREMI C. (2009), Enfermés dehors. Enquêtes sur le confinement des étrangers, Bellecombe-en-Bauges, Éditions du Croquant.

MICHALON B. (2012), «La mobilité au service de l'enfermement? », Géographie et cultures, no. 81, pp. 91-110.

MIGREUROP (2017), Atlas des migrants en Europe. Approches critiques des politiques migratoires, Paris, Armand Colin.

MILHAUD O. (2009), Séparer et punir. Les prisons françaises : mise à distance et punition par l'espace, Université Michel de Montaigne - Bordeaux III.

PAUVROS M. (2014), Les politiques locales d'immigration : un redéploiement des frontières de l'État, Université Paris Diderot - Paris 7.

PILLANT L., TASSIN L. (2015), « Lesbos, l'île aux grillages. Migrations et enfermement à la frontière gréco-turque », Cultures \& Conflits, no. 99-100, pp. 25-55.

RAFFESTIN C. (1986), «Eléments pour une théorie de la frontière », Diogène, no. 134, pp. 3-21.

RITAINE E. (2009), « Des migrants face aux murs d'un monde-frontière », in Jaffrelot C., Lequesne C., L'enjeu mondial. Les migrations, Paris, Presses de Sciences Po, pp. 155-164.

TAZZIOLI M. (2020), « Governing migrant mobility through mobility: Containment and dispersal at the internal frontiers of Europe », Environment and Planning C, vol. 38, no. 1, pp. 3-19.

TRUCCO D. (2018), «7. Prendre en charge et mettre à l'écart. La ville, la frontière et le camp à Vintimille (2015-2017) », in Dubet F., Politiques des frontières, Paris La Découverte, pp. 145-160. 
TYSZLER E., GUENEBEAUD C., PILLANT L. (2019), « Disperser les personnes migrantes », in Barnier S. et al. (dir.), La police des migrants : filtrer, disperser, harceler, Paris, Le passager clandestin, pp. 64-79.

\section{NOTES}

1. Dans le cadre des différents accords bilatéraux signés avec le gouvernement anglais.

2 .

Calais, en 2015 les effectifs de la Police aux frontières sont au nombre de 550. Ils sont répartis entre une division

Transfrontièrequi s'occupe de sécuriser le port et le site du tunnel et une divisionImmigration qui travaille sur la voie publique, les parkings et les lieux de vie des migrants. Dans ses missions, la PAF est soutenue par des Compagnies républicaines de sécurité (CRS) spécialisées dans le maintien de l'ordre qui se relaient toutes les trois semaines. $\mathrm{L}$ eur mission est prioritairement de réaliser des interpellations et de conduire les personnes à

l'hôtel de police. Tous les entretiens sont anonymisés avec un prénom et une initiale inventés. 3. Entretien avec Marc F., ancien sous-préfet de Calais, 12 février 2014

4. Je remercie vivement les auteur.es de l'Atlas des migrants en Europe. Approches critiques des politiques migratoires, et en particulier Olivier Pissoat et Olivier Clochard pour m'autoriser ici à reproduire la carte «Calais : 20 ans d'(in)hospitalité » (Migreurop, $2017: 153$ ).

5. "Contre toute attente, la maire Natacha Bouchart propose de créer un centre pour migrants ", La Voix du Nord, 22/08/14

6. «Exilés et droits fondamentaux : la situation sur le territoire de Calais, Rapport du Défenseur des droits », Octobre 2015, p.11

7. «L'idée ce n'est pas d'évacuer comme on l'a fait jusqu'à présent, je suis allé sur les camps, au squat Blériot, etc. pour leur expliquer, pour faciliter le déménagement », propos du sous-préfet repris dans la presse locale : "Une solution humanitaire sans WC », Nord Littoral, 01/04/15

8. «Le déguerpissement peut se définir comme une expulsion collective et contrainte d'individus qui ne possèdent pas de droits reconnus sur les parcelles qu'ils occupent » (Blot, Spire, 2014). Ce terme est employé principalement dans les pays du Sud et vient au départ de l'administration coloniale pour désigner une entreprise de dépossession des citadins africains de leurs droits sur le sol urbain. Il est aujourd'hui utilisé pour qualifier les déplacements forcés de populations au sein des villes dans une optique de mise à l'écart des populations les plus pauvres du centre-ville et des espaces périphériques valorisés (Blot, Spire, 2014).

9. Le bâtiment de la Socarenam est occupé à plusieurs reprises, malgré des expulsions fréquentes, entre la fermeture du centre de Sangatte en 2002 et sa destruction en 2006.

10. Entretien avec François P., lieutenant CRS, 16 mai 2014

11. Carnet de terrain, Africa House, avril 2010

12. Calais, deux ans après, rapport du réseau No border, 2014.

13. Décision du défenseur des droits ${ }^{\circ}$ MDS 2011-113, le 13 novembre 2012, p.2

14. «Calais, cette frontière tue ", rapport du réseau No border, 2011, p.25

15. Dans le rapport de Human Rights Watch, publié le 20 janvier 2015 et qui s'appuie sur une cinquantaine d'entretiens menés avec des migrants, ceux-ci font notamment le récit de passages à tabac causant parfois des fractures et d'utilisation disproportionnée de gaz lacrymogène.

16. Entretien avec Jacques T., commandant d'une CRS, 16 mai 2014.

17. Décisions du Défenseur des droits n MDS 2011-113, 13 novembre 2012 et nMDS 2014-150, 24 novembre 2014 ; Rapports du Défenseur des droits «Exilés et droits fondamentaux : la situation sur le territoire de Calais », octobre 2015 et "Exilés et droits fondamentaux, trois ans après le rapport Calais », décembre 2018. 
18. Décisions du Défenseur des droits $n^{\circ}$ MDS 2011-113, 13 novembre 2012 ; voir: https:// www.gisti.org/IMG/pdf/jur_ddd_2012-11-13_mds_2011-113.pdf

19. Courrier du directeur de cabinet du ministre de l'Intérieur et du Préfet en réponse au rapport du Défenseur des droits, PN/CMB/N²013_1497_D, 6 mars 2013

20. «Rapport à Monsieur le ministre de l'Intérieur sur la situation des migrants dans le Calaisis : Le pas d'après ", Jean Aribaud et Jérôme Vignon, juin 2015, p.63

21. «Evaluation de l'action des forces de l'ordre à Calais et dans le Dunkerquois », rapport de l'Inspection générale de la police nationale, l'Inspection générale de l'administration et l'Inspection générale de la gendarmerie nationale ", octobre 2017, p.5

22. "Discours d'Emmanuel Macron devant les forces de sécurité à Calais ", 16 janvier 2018. Pour une retranscription du discours, voir: https://www.elysee.fr/emmanuel-macron/2018/01/16/ discours-devant-les-forces-de-securite-a-calais

23. Citation d'un entretien avec un ancien sous-préfet réalisé par Mélanie Pauvros dans son travail de thèse (Pauvros $2014: 328$ ).

24. Volet paysager du permis de construire cité par Berson Emmanuel «Calais ou l'euphémisme d'un mur ", mémoire de master, Université de Lille 1, 2010, p.100

25. Ibid

26. Cette annexe est rendue possible par le vote d'une loi en 2003 qui autorise la construction de salles d'audience à proximité des lieux de rétention.

27. Entretien avec Vincent M., Officier de Police Judiciaire de la PAF, 3 juin 2014

28. "Rapport de visite : centre de rétention administrative de Coquelles (62) », Contrôleur général des lieux de privation de liberté, du 4 au 5 juin 2009. p.27

29. «La loi des jungles : la situation des exilés sur le littoral de la Manche et de la Mer du Nord», Paris, Coordination Française pour le Droit d'Asile, coordination du rapport : Olivier Clochard et Karen Akoka, 2008, p. 69

30. Voir les rapports de la Cimade "Centres et locaux de rétention administrative", puis les rapports conjoints de la Cimade, du Forum des réfugiés, de l'Ordre de Malte et de l'Assfam.

31. Enquête de la Cour des comptes relative à la gestion des centres de rétention administrative, juillet 2009.

32.

Selon le règlement Dublin III de 2013, un seul État membre de l'UE est responsable de la demande d'asile. Est responsable de la demande le pays où il existe des traces d'entrée ou de séjour de la personne, ou le pays où est déjà présent un.e membre, réfugié.e ou demandeur.euse d'asile, de sa

famille. La mise en place en 2004 du système d'Eurodac, une base de données qui contient les empreintes à la fois des demandeurs.euses d'asile, des réfugié.es et des personnes interpellées lors d'un franchissement illégal d'une frontière, tente de rendre opérationnel le règlement de

Dublin.

33. Une réadmission Schengen est prise à l'encontre d'une personne qui dispose de la preuve d'un séjour ou d'une entrée régulière dans un État membre de l'espace Schengen ou s'il provient directement d'un de ces États (titre de transport, personne trouvée dans un camion en provenance de la Belgique).

34. «Centres et locaux de rétention administrative ", rapport 2014, Cimade, Forum des réfugiés, Ordre de Malte, Assfam.

35. Chiffres donnés par Anne de Loisy, voir: «Dans la jungle des clandestins », Politis, $n^{\circ} 929$, $14 / 12 / 2006$

36. "Main basse sur l'asile. Le droit d'asile (mal)traité par les préfets", Cimade, Rapport d'observation, juin 2007, p. 38.

37. Entretien avec François P., lieutenant CRS, 16 mai 2014 
38.

Circulaire du 20 novembre 2015 du ministère de l'Intérieur et du ministère du Logement relative à la création de centres de mise à l'abri pour les migrants de Calais. 39. Suite au contrôle du CRA de Coquelles en juillet 2015, le contrôleur général des lieux de privation de liberté adresse des recommandations pour que s'arrêtent les déplacements collectifs de personnes étrangères interpellées à Calais. Il rend publique ces recommandations quelques mois plus tard: https://www.cglpl.fr/wp-content/uploads/2017/06/Rapport-de-ladeuxi\%C3\%A8me-visite-du-centre-de-r\%C3\%A9tention-administrative-de-Coquelles.pdf

40. « Migrants renvoyés par avion : c'est 4000 euros l'heure de vol », Nord Littoral, 11/09/2015

41. «Recommandations en urgence du Contrôleur général des lieux de privation de liberté du 13 novembre 2015 relatives aux déplacements collectifs de personnes étrangères interpellées à Calais », Journal officiel de la République Française, 2 décembre 2015.

42. Entretien avec Jérôme F., détaché syndical permanent sur le Pas-de-Calais, PAF, 23 mai 2014

43. Entretien avec Jacques T., commandant d'une CRS, 16 mai 2014

44. Entretien avec Didier P., lieutenant chargé de la communication à la Direction centrale des CRS de Paris, 28 mars 2014

45. " Déplacer le problème, ça me va très bien! ", Métronews, 17/09/2009

46. Résumé du rapport « La loi des jungles », 2008.

47. Expression employée par Olivier Milhaud dans sa thèse Séparer et Punir. Les prisons françaises : mise à distance et punition par l'espace (Milhaud, 2009 : 198) à propos des stratégies de distanciation mises en place des riverain.es vivants proches de prisons.

\section{RÉSUMÉS}

La ville de Calais, un des principaux espaces de transit vers l'Angleterre, a été le théâtre depuis plus de vingt ans d'une sécurisation croissante de son port et du site d'entrée du tunnel sous la Manche afin de filtrer et de bloquer les traversées des personnes migrantes. Au-delà de ces politiques d'immobilisation, les stratégies de contrôle des pouvoirs publics français passent également par une mise en mouvement contrainte des personnes migrantes. En axant le propos sur les pratiques policières de déplacements forcés, cet article souhaite contribuer à l'analyse des dispositifs spatiaux de maintien de l'ordre en contexte frontalier. Il s'appuie, pour ce faire, sur une enquête de terrain menée à Calais entre 2010 et 2015 auprès des acteurs du contrôle migratoire.

The city of Calais, one of the main transit areas to England, has for more than twenty years been the scene of reinforced security measures in its port and at the entrance site of the Channel Tunnel to filter and block the crossings of migrants. In addition to these immobilization policies, the strategies of the French public authorities also involve the forced mobility of migrants. By focusing on police practices of forced displacement, this paper aims to contribute to the analysis of spatial policing mechanisms in border contexts. It is based on a field survey conducted in Calais between 2010 and 2015 among actors involved in migration control. 
INDEX

Thèmes: Carnets de recherches

Keywords : Police, Forced Mobility, Migration, Calais

Mots-clés : police, mobilités forcées, migration, Calais

\section{AUTEUR}

CAMILLE GUENEBEAUD

Maître de Conférences en géographie à l'Université Paris 8 Vincennes-Saint-Denis.

camille.guenebeaud[at]gmail.com 